\title{
PROPRIEDADES ANTIOXIDANTES DE COMPOSTOS FENÓLICOS
}

\section{ANTIOXIDANTS PROPERTIES OF PHENOLIC COMPOUNDS}

\author{
DEGÁSPARI, Cláudia Helena'; WASZCZYNSKYJ, Nina² \\ ${ }^{1}$ Universidade Tuiuti do Paraná (Doutoranda), e-mail: claudia.degaspari@utp.br \\ 2 Universidade Federal do Paraná (Doutora), e-mail: ninawas@ufpr.br
}

Recebido em: 01/2004

Aprovado em: 02/0404

RESUMO

Os organismos vivos estão constantemente sujeitos à ação oxidativa do oxigênio, sendo que diversos estudos têm demonstrado que o consumo de substâncias antioxidantes na dieta diária, pode produzir uma ação protetora efetiva contra estes processos oxidativos que ocorrem no organismo. Foi descoberto que uma série de doenças entre as quais câncer, aterosclerose, diabetes, artrite, malária, AIDS, doenças do coração, podem estar ligadas aos danos causados por formas de oxigênio extremamente reativas denominadas de "substâncias reativas oxigenadas" ou ROS. Estas substâncias também estão ligadas com processos responsáveis pelo envelhecimento do corpo. Os produtos alimentícios também se mostram suceptíveis a estes processos oxidativos, resultando em substâncias finais prejudiciais ou com características sensoriais indesejáveis, reduzindo com isso o prazo de validade dos produtos. A partir do início dos anos 80, o interesse em encontrar antioxidantes naturais para o emprego em produtos alimentícios ou para uso farmacêutico, tem aumentado consideravelmente, com o intuito de substituir antioxidantes sintéticos, os quais têm sido restringidos devido ao seu potencial de toxicidade. O presente trabalho procurou realizar uma revisão bibliográfica sobre os principais pontos que abrangem as propriedades antioxidantes de compostos fenólicos, entre eles os flavonóides.

Palavras-chave: antioxidantes; compostos fenólicos; flavonóides

\section{ABSTRACT}

The alive organisms are constantly subjects to the oxygen oxidative action, and several studies have been demonstrating that the consumption of antioxidant substances in the daily diet, it can produce an effective protecting action against these oxidatives processes that happen in the organism. It was discovered that a series of diseases among the ones which cancer, aterosclerose, diabetes, arthritis, malaria, AIDS, heart diseases, can be linked to the damages caused by oxygen extremely reactive forms denominated by "reactivate oxygenated substances" or ROS. These substances are also responsible for processes of aging. The food products are also sensible to these oxidatives processes, resulting in harmful final substances or with undesirable sensorial characteristics, and reducing the products shelf-life. After the 80 years, the interest by finding natural antioxidants for the employment in food products or for pharmaceutical use, it has been increasing considerably, trying to substitute the synthetic antioxidants, which have been restricted due to its toxicicological potential. The present work tried to accomplish a review of the main points that envolve the antioxidant properties of phenolics compouds, among them the flavonols.

Key-words: antioxidants; flavonols; phenolics compounds

\section{INTRODUÇÃO}

Os processos de oxidação de substâncias orgânicas são uma das principais causas da redução da vida de prateleira dos produtos alimentícios industrializados bem como das matérias-primas em geral. Portanto, o conhecimento e compreensão dos mecanismos de reação e as formas de controle para os mesmos são de suma importância econômica para a indústria alimentícia.

Dentre as principais reações de oxidação em produtos alimentícios se destacam:

- escurecimento enzimático e

- oxidação de lipídios.

No caso das reações de oxidação de lipídios, os principais problemas decorrentes são as alterações sensoriais envolvendo o desenvolvimento de notas aromáticas desagradáveis, 
denominadas de uma forma generalizada de ranço. Estas reações ocorrem com substratos específicos que são os ácidos graxos, encontrados normalmente na constituição dos glicerídios. Esta alteração na qualidade de um produto é o principal parâmetro de controle físico-químico (ponto crítico de controle) que define o prazo de validade de diversos produtos alimentícios processados, principalmente quando os mesmos apresentam valores de atividade de água inferiores a 0,3. Nesta faixa de valores de atividade de água, atinge-se a zona de adsorsão primária ou a monocamada, que propicia a ação catalítica de metais, favorecendo o desenvolvimento das reações de oxidação dos lipídios. (BOBBIO \& BOBBIO, 1992; FENNEMA, 1993)

\section{OXIDAÇÃO DE LIPÍDIOS}

Os mecanismos de reação para explicar a ocorrência destes processos de deterioração em lipídios ainda não estão totalmente esclarecidos. Sabe-se, no entanto que, os mesmos podem se oxidar por meio de mecanismos via enzimática ou não enzimática.

Os alimentos contendo significantes teores de ácidos graxos polinsaturados têm contribuído pela necessidade de se empregar agentes antioxidantes para prevenir a oxidação. Os antioxidantes mais largamente empregados são o butil-hidroxi-tolueno (BHT), butil-hidroxi-anisol (BHA) e butilhidroquinonas terciárias e em alguns casos a ação sinérgica do ácido cítrico é indispensável. Estes agentes antioxidantes tendem a estabilizar os ácidos graxos em alimentos através da reação com radicais livres, quelando íons metálicos e interrompendo a fase de propagação da oxidação lipídica. Os antioxidantes naturais ou artificiais apresentam funções similares, sendo que vem sendo questionada a salubridade de alguns antioxidantes comerciais, visto que alguns estudos têm demonstrado que os mesmos podem favorecer efeitos mutagênicos e carcinogênicos. (BIRCH et al., 2001)

\section{AGENTES ANTIOXIDANTES}

Antioxidantes são compostos que podem retardar ou inibir a oxidação de lipídios ou outras moléculas, evitando o início ou propagação das reações em cadeia de oxidação. A atividade antioxidante de compostos fenólicos é principalmente devida às suas propriedades de óxido-redução, as quais podem desempenhar um importante papel na absorção e neutralização de radicais livres, quelando o oxigênio triplete e singlete ou decompondo peróxidos. Em geral, existem duas categorias básicas de antioxidantes: os naturais e os sintéticos. (ANTUNES \& CANHOS, 1984; BRENNA \& PAGLIARINI, 2001; ZHENG \& WANG, 2001; FENNEMA, 1993; SIMÃO, 1985).

Diversos estudos têm demonstrado que o consumo de substâncias antioxidantes na dieta diária, pode produzir uma ação protetora efetiva contra os processos oxidativos que naturalmente ocorrem no organismo. Foi descoberto que uma série de doenças entre as quais câncer, aterosclerose, diabetes, artrite, malária, AIDS, doenças do coração, podem estar ligadas aos danos causados por formas de oxigênio extremamente reativas denominadas de "substâncias reativas oxigenadas" ou simplesmente ROS. Estas substâncias também estão ligadas com processos responsáveis pelo envelhecimento do corpo. (BRENNA \& PAGLIARINI, 2001; YILDRIM, MAVI \& KARA, 2002)

Os mecanismos endógenos de defesa (ou mediadores de redox tais como: superóxido dismutase, catalase, peroxidase e metaloproteínas) podem ser auxiliadas favoravelmente 
com a introdução de antioxidantes por meio da dieta. (BRENNA \& PAGLIARINI, 2001; YILDRIM, MAVI \& KARA, 2002).

As ROS's são, na verdade, as várias formas de oxigênio ativado (singlete), entre as quais se incluem os denominados radicais livres. Nos organismos vivos as várias formas de ROS's podem se constituídos de diversas maneiras. Por exemplo, nas fontes exógenas produtoras de radicais livres inclui-se a fumaça do tabaco, radiações ionizantes, solventes orgânicos e pesticidas. (YILDRIM, MAVI \& KARA, 2002)

Da mesma forma, o emprego de agentes antioxidantes visando o aumento do prazo de validade de produtos alimentícios é uma constante na área da tecnologia de alimentos. A Resolução n ${ }^{\circ} 04$ de 24/1 1/88 relaciona um total de 13 aditivos permitidos classificados como antioxidantes, possíveis de serem adicionados em aproximadamente 40 a 50 alimentos. (MINISTÉRIO DA SAÚDE, 1988)

A partir do início dos anos 80, o interesse em encontrar antioxidantes naturais para o emprego em produtos alimentícios ou para uso farmacêutico, tem aumentado consideravelmente, com o intuito de substituir antioxidantes sintéticos, os quais tem sido restringidos devido ao seu potencial de carcinogênese, bem como pela comprovação de diversos outros males como: aumento do peso do fígado e significativa proliferação do retículo endoplasmático. (MELO \& GUERRA, 2002; SIMÃO, 1985; YILDRIM, MAVI \& KARA, 2002; ZHENG \& WANG, 2001).

As matérias-primas in natura disponíveis como: frutas, vegetais em geral e condimentos contém numerosos fitoquímicos além dos compostos fenólicos como por exemplo compostos nitrogenados, carotenóides, ácido ascórbico e tocoferóis. Muitos destes fitoquímicos apresentam significante capacidade antioxidante e são associados à baixa incidência e baixa mortalidade de câncer em seres humanos. (BIRCH et al., 2001; SLUIS et al., 2001; TOMÁS-BARBERÁN el al., 2001; VINSON et al., 2001; WANG \& ZHENG, 2001; WATANABE, 1998; YILDRIM, MAVI \& KARA, 2002; ZHENG \& WANG, 2001)

\section{COMPOSTOS FENÓLICOS DE FONTES VEGETAIS}

Os compostos fenólicos de fontes vegetais podem se divididos em dois grupos: os flavonóides e os não flavonóides, sendo que ambos são metabólitos secundários presentes em frutas e vegetais. Os denominados de flavonóides são os que apresentam a estrutura química descrita como C6-C3-C6. Já os denominados de não flavonóides são classificados como: (MELO \& GUERRA, 2002; BURNS et al., 2001)

- os derivados das estruturas químicas $\mathrm{C}_{6}-\mathrm{C}_{1}$ específicas dos ácidos hidroxi benzóico, gálico e elágico;

- os derivados das estruturas químicas $\mathrm{C}_{6}-\mathrm{C}_{3}$ específicas dos ácidos cafêico e p-cumárico hidroxi cinamatos e

- os derivados das estruturas químicas $\mathrm{C}_{6}-\mathrm{C}_{2}-\mathrm{C}_{6}$ específicas do trans resveratrol, cis-resveratrol e trans-resveratrol-glucosídio.

A distribuição dos flavonóides nos vegetais depende de diversos fatores de acordo com a fila/ordem/família do vegetal, bem como da variação das espécies. Os flavonóides são formados da combinação de derivados sintetizados da fenilalanina (via metabólica do ácido chiquímico) e ácido acético. Os padrões de distribuição dependem do grau de acesso à luminosidade, especialmente raios ultravioleta B, pois a formação dos flavonóides é 
acelerada pela luz. Consequentemente, plantas cultivadas em estufas, onde os raios ultravioleta são bloqueados o conteúdo de flavonóides é reduzido. Vegetais que crescem na Espanha ou na África do Sul são apontados como contendo de 4 a 5 vezes mais flavonóides que os que crescem no Reino Unido. (AHERNE \& O'BRIEN, 2002; BOBBIO \& BOBBIO, 1989; BURNS et al., 2001; FENNEMA, 1993; SELLAPPAN, AKOH \& KREWER, 2002).

O grupo dos flavonóides é também conhecido como polifenólicos e geralmente ocorrem em plantas na forma de glucosídios, sendo uma das substâncias responsáveis pela atribuição do perfil sensorial de frutas, atribuindo-lhes o corpo característico. Mais de 6.000 diferentes estruturas já foram identificadas e este número continua a aumentar. (AHERNE \& O'BRIEN, 2002; BOBBIO \& BOBBIO, 1989; CORDENUNSI et al., 2002; FENNEMA, 1993; SLUIS et al., 2001)

Em pesquisas epidemiológicas alguns flavonóides apresentam-se associados com a proteção contra doenças do envelhecimento. Isto pode ser justificado devido à sua ação antioxidante. A formação de radicais livres pelo oxigênio é supostamente a chave para o desenvolvimento de câncer e doenças coronárias, aliado à função protetora da membrana celular. Radicais livres podem atacar biomoléculas, dentre as quais destacam-se os lipídios, as proteínas ou DNA propriamente dito, os quais podem ser preservados pela ação dos antioxidantes. (BIRCH et al., 2001; BRENNA \& PAGLIARINI, 2001; KOO \& SUHAILA, 2001; SELLAPAN, AKOH \& KREWER, 2002; SLUIS et al., 2001; VINSON et al., 2001; YILDRIM, MAVI \& KARA, 2002; ZHENG \& WANG, 2001; WANG \& ZHENG, 2001)

As frutas, principalmente as que apresentam a coloração vermelha/azul, são as mais importantes fontes de compostos fenólicos em dietas alimentares. Especialmente os derivados do ácido hidroxibenzóico e do ácido hidroxicinâmico dentre estes cita-se: as antocianinas, os flavonóis, as catequinas e os taninos (hidrolisados ou condensados) nas quais estão freqüentemente presentes. Muitos destes compostos apresentam uma grande gama de efeitos biológicos, incluindo ações antioxidantes, antimicrobiana, anti-inflamatória e vasodilatadora. Estes compostos fenólicos apresentam diversas funções de defesa para as plantas, não somente contra agentes do meio ambiente (luz, temperatura e umidade), mas para fatores internos incluindo diferenças genéticas, nutrientes, hormônios, contribuindo para a sua síntese. (AHERNE \& O'BRIEN, 2002; BURNS et al., 2001; KÄHKÖNEN, HOPIA \& HEINONEN, 2001; SELLAPAN, AKOH \& KREWER, 2002; SLUIS et al., 2001; ZHENG \& WANG, 2001).

Sob o ponto de vista nutricional, os flavonóides são reconhecidamente agentes antioxidantes capazes de inibir a oxidação de lipoproteínas de baixa densidade LDL), além destes reduzirem significativamente as tendências a doenças trombóticas (RAUHA et al., 2000)

Os efeitos bioquímicos e farmacológicos dos flavonóides são muito vastos, dentre estes destacam-se as ações antioxidante, antiinflamatória e antiplaquetária, alem de efeitos antialergênicos. Eles podem inibir enzimas destacando-se: a prostaglandina sintetase, a lipoxigenase e a ciclooxigenase, todas relacionadas diretamente com a tumorogênise. Também tem poder de induzir enzimas do sistema desentoxicante como a glutationa Stransferase. Quando em alimentos, os flavonóides agem de forma a poupar o consumo de vitamina C, evitando a formação de radicais livres. (KOO \& SUHAILA, 2001)

5 COMPOSTOS FENÓLICOS TIPO FLAVONÓIDES

A estrutura dos flavonóides está baseada no núcleo flavilium, o qual consiste de três 
anéis fenólicos. O benzeno do primeiro anel é condensado com o sexto carbono do terceiro anel que na posição 2 carrega um anel de fenil-benzeno como substituinte. $O$ terceiro anel pode ser um pirano heterocíclico, gerando as estruturas básicas das leucoantocianinas (ou proantocianinas ou catequinas) e as antocianidinas, denominado de núcleo flavan. No caso do terceiro anel apresentar como uma pirona, ocorre a formação das flavonas, flavonóis, flavanonas, isoflavonas, chalconas e auronas, recebendo a denominação de núcleo 4oxo-flavonóide. (AHERNE \& O'BRIEN, 2002)

Exceto o grupo das leucoantocianinas, os demais flavonóides ocorrem em plantas sempre acompanhadas por glicídios, que recebe a denominação de glico-flavonóide. Quando se apresentam isentos de glicídios, a estrutura recebe o nome de aglicona. (AHERNE \& O'BRIEN, 2002)

No caso dos taninos, os mesmos são classificados em dois grupos principais, cujas estruturas são muito diferentes entre si, embora todos tenham molécula poli-hidroxifenóis ou seus derivados. Os pertencentes ao primeiro grupo são denominados taninos hidrolisáveis, que incluem os galitaninos e os elagitaninos, polímeros derivados dos ácidos gálico e elágico. Este grupo de taninos é comumente utilizado para a curtição de couros. (BOBBIO \& BOBBIO, 1989; FENNEMA, 1993; MELO \& GUERRA, 2002)

O outro tipo de taninos é denominado de taninos condensados e são encontrados em maior quantidade e de maior importância em alimentos. Apresentam uma estrutura semelhante aos flavonóides, com coloração variando do vermelho ao marrom. A presença de pequenas quantidades de taninos em frutos confere-Ihes características sensoriais desejáveis, ditas como "o corpo da fruta". No entanto, quantidades maiores conferem aos frutos e outros alimentos características adstringentes. A sensação de adstringência é gerada devido à propriedade que os taninos apresentam de precipitar proteínas. Quando em contato com as proteínas da saliva, forma um complexo insolúvel que popularmente se caracteriza pela sensação "amarrando a língua". (BOBBIO \& BOBBIO, 1989)

\subsection{ANTOCIANINAS}

As antocianinas são um grupo de pigmentos vegetais hidrosolúveis, amplamente distribuídos no reino vegetal. Seu espectro de cor vai do vermelho ao azul, apresentando-se também como uma mistura de ambas as cores resultando em tons de púrpura. Muitas frutas, hortaliças, folhas e flores devem sua atrativa coloração a estes pigmentos que se encontram dispersos nos vacúolos celulares.

Atualmente se conhece 20 antocianinas, mas apenas 6 delas são importantes na tecnologia de alimentos: pelargonidina, cianidina, delfinidina, peonidina, petunidina $e$ malvidina. As demais são relativamente raras e são normalmente encontradas em flores e folhas. (FENNEMA, 1993)

O principal interesse das antocianinas na tecnologia de alimentos se refere à conferência de coloração adequada e desejada. No entanto, como trata-se de um íon, o cátion flavilium se mostra muito reativo. As reações decorrentes em geral resultam na descoloração do pigmento e quase sempre são indesejáveis no processamento de frutas e hortaliças. O pré processamento de branqueamento é um dos principais responsáveis pela perda das antocianinas nos alimentos, principalmente se ele é acompanhado da adição de sulfitos ou dióxido de enxofre. A adição destas substâncias resulta em uma descoloração rápida das antocianinas, que tornam-se amareladas e, portanto, descaracterizadas. 


\subsection{ANTOXANTINAS}

O caráter fenólico das antoxantinas confere a esses flavonóides a capacidade de seqüestrar metais (agentes quelantes), fazendo com que possam ter função antioxidante em óleos e gorduras. (BOBBIO \& BOBBIO, 1992).

Pelo seu possível valor antioxidante de óleos e gorduras, os flavonóides têm despertado interesse em seu caráter polifenólico dos flavonóides e sua capacidade para seqüestrar metais. No entanto, sua limitada solubilidade em meio oleoso limita seu emprego. (FENNEMA, 1993)

Os flavonóis quercetina, caferol e miricetina se apresentam em grandes quantidades no chá preto em pó, sendo os responsáveis pela sua adstringência. A rutina forma um complexo com o ferro produzindo uma coloração escura em aspargos enlatados e com estanho uma coloração amarela. (FENNEMA, 1993)

As isoflavonas ou fitoestrógenos são encontrados no grão de soja, brotos de alfafa, sementes de linhaça, trevo vermelho e outros vegetais. Sua maior concentração é observada no gérmen dos grãos de soja. Estes compostos apresentam as atividades antioxidantes, bactericida, antifûngica, anticarcinogênica, antinflamatória, antimutagênica, antihipertensiva, antiviral, antiproliferativa, estrogênica e às vezes antiestrogênica (FERREIRA, 2002).

O consumo de soja e subprodutos derivados, faz com que as isoflavonas presentes sejam hidrolisadas por glicosidases intestinais, liberando suas formas biologicamente ativas: agliconas, daidzeína, genísteina e glicíteína, todas com efeitos benéficos para o organismo humano. Os efeitos relacionados à atividade estrogênica ( 100 vezes inferior à do b-estradiol) foram confirmados por diversos estudos. Em presença de estrogênios humanos, as isoflavonas funcionam como antiestrógenos, competindo com os hormônios por seus receptores nas células-alvo, e evitando que estes exerçam seus efeitos negativos. Na ausência ou insuficiência daqueles (menopausa) apresentam efeito estrogênico, substituindo-os e aliviando os sintomas indesejáveis da menopausa, tais como risco de desenvolvimento de doenças cardiovasculares, Diabetes Mellitus, aterosclerose e osteoporose. Assim, as isoflavonas vêm despertando atenção de mulheres climatéricas para as quais a terapia de reposição hormonal é desaconselhável (PARK et al., 2001).

\section{COMPOSTOS FENÓLICOS TIPO NÃO FLAVONÓIDES}

Os ácidos fenólicos estão reunidos em dois grupos, a saber: derivados do ácido hidroxicinâmico e derivados do ácido hidroxibenzóico. Os derivados do ácido hidroxicinâmico são compostos fenólicos de ocorrência natural que possuem um anel aromático com uma cadeia carbônica, constituída por 3 carbonos ligada ao anel. Os ácidos p-cumárico, ferúlico, caféico e sináptico são os hidroxicinâmicos mais comuns na natureza. Estes ácidos existem nas plantas, usualmente na forma de ésteres, a exemplo do ácido clorogênico, éster do ácido quínico, cuja molécula é constituída pelo ácido quínico esterificado ao ácido caféico. Tambérn são encontrados na forma de glicosídeos ou ligados a proteínas e a outros polímeros da parede celular e, raramente, como ácidos livres. Isômeros do ácido clorogênico e do ácido caféico são descritos com antioxidantes (BELITZ \& GROSCH, 1988; DURÁN \& PADILLA, 1993; HARBORNE, 1973).

No grupo dos ácidos hidroxibenzóicos, compostos que possuem grupo carboxílico 
ligado ao anel aromático, destacam-se os ácidos protocatecuíco, vanílico, siríngico, gentísico, salicílico, elágico e gálico. Esses dois grupos de ácidos fenólicos têm apresentado propriedades antioxidantes (HARBORNE, 1973). Embora outras características também contribuam para a atividade antioxidante dos ácidos fenólicos e seus ésteres, esta é, geralmente, determinada pelo número de hidroxilas presentes na molécula (RAJALAKSMI \& NARASIMHAN, 1995).

A hidroxila do ácido ferúlico existente na posição orto com o grupo metoxila, doador de elétrons, é um fator que aumenta a estabilidade do radical fenoxil e aumenta a eficiência antioxidante do composto (CUVELIER, RICHARD \& BERSET, 1992). A presença de uma segunda hidroxila na posição orto ou para, também aumenta a atividade antioxidante. O ácido caféico, que apresenta essa característica, possui uma atividade antioxidante maior do que o ácido ferúlico (CHEN \& HO, 1997). O efeito seqüestrante do radical hidroxil parece estar diretamente relacionado aos grupos hidroxil localizados na posição para no anel aromático.

Os ácidos sináptico, ferúlico e p-cumárico são antioxidantes mais ativos do que os derivados do ácido benzóico, tais como ácido procatecuíco, siríngico e vanílico. Isso se deve à dupla ligação presente na molécula dos derivados do ácido cinâmico $(-\mathrm{HC}=\mathrm{CH}$ $\mathrm{COOH})$, que participa da estabilização do radical por ressonância de deslocamento do elétron desemparelhado, enquanto que os derivados do ácido benzóico não apresentam essa característica (WANASUNDARA, AMAROWICZ \& SHAHIDI, 1994).

\section{CONCLUSÃO}

Dentre tudo o que foi exposto, verifica-se que as pesquisas envolvendo agentes antioxidantes em espécies vegetais devem continuar, pois as mesmas se mostram de suma importância tanto para a indústria alimentícia quanto para a indústria farmacêutica. No que diz respeito ao ramo alimentício, as pesquisas de agentes antioxidantes se mostram importantes no sentido de obter aditivos alimentícios com menos efeitos colaterais possíveis, características indesejáveis estas que são observadas nos atuais antioxidantes empregados em produtos alimentícios. Já no ramo farmacêutico a busca é por substâncias de excelência quanto aos aspectos funcionais no combate aos radicais livres e todos os possíveis males que podem causar à saúde humana.

\section{REFERÊNCIAS BIBLIOGRÁFICAS}

AHERNE, S.A.; O'BRIEN, N.M. Dietery flavonols: chemistry, food content, and, metabolism. Nutrition. New York: v. 18, n. 1, p. 75-81, 2002.

ANTUNES, A. J.; CANHOS, V. Aditivos em Alimentos. Campinas: Editora da UNICAMP, 1984.

BELITZ, H.D.; GROSCH, W. Química de los alimentos. Zaragoza: Acribia, p. 645- 656, 1988.

$\mathrm{BIRCH}, \mathrm{A} . \mathrm{E}$. et al. Antioxidant proprieties of evening primrose seed extracts. J. Agric. Food Chemistry, Chicago: v.49, p. 4502-4507, 2001.

BOBBIO, F.O.; BOBBIO, P. A. Introdução à química de alimentos. 2.ed. São Paulo : Varela, 1989.

BOBBIO, P. A. ; BOBBIO, F. O. Química do processamento de alimentos. 2.ed. São Paulo : Varela, 1992.

BRENNA, O.V.; PAGLIARINI, E. Multivariate analyses of antioxidant power and polyphenolic composition in red wines. J. Agric. Food Chemistry. Chicago: v.49, p. 4841-4844, 2001.

BURNS, J. et. al. Extraction of phenolics and changes in antioxidant activity of red wines during vinification. J. Agric. Food Chemistry. Chicago: v.49, p. 5797-5808, 2001.

CHEN, J.H.; HO, C.T. Antioxidant activities of acid caffeic and its related hydroxycinnamic acid compounds. J. Agric. Food Chemistry. Chicago: v, 45, n. 7, p. 2374-2378, 1997.

CORDENUNSI, B.R. et. al. Influence of cultivar on quality parameters and chemical composition of strawberry fruits grown in Brazil. J. Agric. Food Chemistry. Chicago: v. 50, n. 9, p. 2581-2586, 2002. 
CUVELIER, M.E.; RICHARD, H.; BERSET, C. Comparison of antioxidative activity of some acid-phenols; structureactivity relationship. Bioscience, Biotechnology and Biochemistry. Tokyo: v. 56, n. 2, p. 324-235, 1992.

DURÁN, R.M.; PADILLA, R.B. Actividad antioxidante de los compuestos fenólicos. Grasas y Aceites. Sevilla: v. 44, n. 2, p. 101-106, 1993.

FENNEMA, O.R. Química de los alimentos. 2.ed. Zaragoza: Acribia, 1993.

FERREIRA, R.A.S. Dosiê soja. Nutrição Brasil. Rio de Janeiro: v. 1, n.3, p. 177-186, 2002.

HARBORNE, J.B. Phytochemical Methods. London: Chapman and Hall, p. 33-88, 1973.

KÄHKÖNEN, M.P.; HOPIA A.I.; HEINONEN, M. Berry phenolics and their antioxidant activity. J. Agric. Food Chemistry. Chicago: v.49, p. 4076-4082, 2001.

KOO, H.M.; SUHAILA, M. Flavonoid (myricetin, quercetin, kaempferol, luteolin, and apigenin) content of edible tropical plants. J. Agric. Food Chemistry. Chicago: v.49, n. 6, p. 3106-3112, 2001.

MELO, E.A.; GUERRA, N.B. Ação antioxidante de compostos fenólicos naturalmente presentes em alimentos. Bol. SBCTA. Campinas: v.36, n. 1, p. 1-11, 2002.

MINISTÉRIO DA SAÚDE. Resolução, $\mathbf{n}^{\circ} 04$ de 24 de novembro de 1988. Aditivos Intencionais. Brasília: Ministério da Saúde, 1988.

PARK, Y.K. et al. Biotransformação de isoflavonas de soja. Biotecnologia, Ciência e Desenvolvimento. Brasília: $n$. 20, p. 12-14, 2001.

PARK, Y.K.; KOO, M.H.; CARVALHO, P.O. Recentes progressos dos alimentos funcionais. Boletim da SBCTA, v. 31 , n. 2 , p. 200-206, 1997.

POKORNÝ, J. Natural antioxidants for food use. Trends in Food Science and Technology. Cambridge: v. 2, n. 9, p. 223-227, 1991.

RAJALAKSMI, D.; NARASIMHAN, S. Food antioxidants: sources and methods of evalution. In: MADHAVl, D.L.; DESHPANDE, S.S.; SALUNKHE, D.K. Food Antioxidants - technological, toxicological and health perspectives. New York: Marcel Dekker, p. 65-157, 1995.

RAUHA, J.P. et. al. Antimicrobial effects of Finnish plant extracts containing flavonoids and other phenolic compounds. International Journal of Food Microbiology. Amsterdam: v.56, n. 1, p. 3-12, 2000.

SELLAPPAN, S.; AKOH, C.C.; KREWER, G. Phenolic compounds and antioxidant capacity of Georgia-grown blueberries and blackberries. J. Agric. Food Chemistry, Chicago: v.50, n. 8, p. 2432-2438, 2002.

SIMÃO, A.M. Aditivos para alimentos sob o aspecto toxicológico. São Paulo: Nobel, 1985.

SLUIS, A.A. et al. Activity and concentration of polyphenolic antioxidants in apple: effect of cultivar, harvest year, and storage conditions. J. Agric. Food Chemistry. Chicago: v.49, p. 3606-3613, 2001.

TOMÁS-BARBERÁN, F.A. et al. HPLC-DAD-ESIMS analysis of phenolic compounds in nectarines, peaches, na plums. J. Agric. Food Chemistry. Chicago: v.49, p. 4748-4760, 2001.

VINSON, J.A. et al. Phenol antioxidant quantity and quality in foods: fruits. J. Agric. Food Chemistry. Chicago: v.49, p. 5315-5321, 2001.

WANASUNDARA, U.; AMAROWICZ, R.; SHAHIDI, F. Isolation and identification of an antioxidative component in canola. J. Agric. Food Chemistry. Chicago: v. 42, n. 6, p. 1285-1290, 1994.

WANG, S.Y.; ZHENG, W. Effect of plant growth temperature on antioxidant capacity in strawberry. J. Agric. Food Chemistry. Chicago: v.49, p. 4977-4982, 2001.

WATANABE, M. J. Catechins as antioxidants from buckwheat (Fagopyrum esculentum Moech). J. Agric. Food Chemistry. Chicago: vol. 46, no. 3, p. 839-845, 1998.

YILDIRIM, A.; MAVI, A.; KARA, A.A. Determination of antioxidant and antimicrobial activities of Rumex crispus $L$. extracts. J. Agric. Food Chemistry. Chicago: v.49, p. 4083-4089, 2001.

ZHENG, W.; WANG, S.Y. Antioxidant activity and phenolic compounds in selected herbs. J. Agric. Food Chemistry. Chicago: v.49, p. 5165-5170, 2001. 